\title{
Formação, Treinamento e Aperfeiçoamento em Oncologia Infantojuvenil no Âmbito do Programa Nacional de Apoio à Atenção Oncológica:um Panorama do Triênio 2016-2018
}

doi: https://doi.org/10.32635/2176-9745.RBC.2018v64n3.32

Formation, Training and Improvement in Youth Oncology in the Scope of the National Program of Support to Cancer Oncology: a Panorama of the Triennium 2016-2018

Formación, Entrenamiento y Perfeccionamiento en Oncología Infantil y Juvenil en el Marco del Programa Nacional de Apoyo a la Atención Oncológica: un Panorama del Trienio 2016-2018

\section{Vanessa Murta Rezende'; Gabriela Furtado Neves²}

\section{Resumo}

Introduçáo: O Programa Nacional de Apoio à Atenção Oncológica (Pronon) é fruto de uma parceria entre os Ministérios da Saúde e da Fazenda, que, conjuntamente, determinam um valor global de renúncia fiscal a ser empregada a cada período. Entre os temas prioritários do programa, tem-se a oncologia infantojuvenil e é possível a submissão de projetos, pelas instituições de saúde do Terceiro Setor, nas áreas de assistência, pesquisa e formação profissional. Objetivo: Apresentar um panorama do triênio 2016-2018, referente aos projetos voltados para essa temática, na área de formação, treinamento e aperfeiçoamento de recursos humanos. Método: Estudo de caso e análise qualiquantitativa, tendo como amostra todos os projetos apresentados ao Ministério da Saúde, no âmbito do Pronon, no período. Resultados: Foi possível observar que o número de projetos apresentados na esfera do Pronon, na temática de oncologia infantojuvenil, é muito baixo, representando $14,28 \%$ do total de projetos expostos no triênio e, destes, somente um foi aprovado. Conclusáo: Conclui-se que há uma carência de projetos tanto apresentados como aprovados envolvendo essa temática. Dessa forma, busca-se incentivar as instituições do Terceiro Setor que atuam na prestação de serviços oncológicos a participarem do programa por meio de projetos com capacidade técnica operacional, considerados de relevância para o Sistema Único de Saúde e seus usuários.

Palavras-chave: Oncologia; Programas Nacionais de Saúde; Capacitação de Recursos Humanos em Saúde; Pediatria.

\begin{abstract}
Introduction: The National Program of Support to Cancer Oncology (Pronon) is the result of a partnership between the Ministries of Health and of Finance, which together determine a global amount of tax relief to be used each cycle. Among the priority themes of the program are the youth oncology and it is possible to submit projects by the Health Institutions of the Third Sector in the areas of assistance, research and professional training. Objective: Present a panorama of the triennium 2016-2018 referring to the projects focused on this theme, in the area of training, training and improvement of human resources. Method: Study and qualitative and quantitative analysis were done, taking as a sample all the projects submitted to the Ministry of Health, within Pronon, in the period. Results: It was possible to observe that the number of projects presented in the scope of Pronon in the subject of youth oncology is very low, representing $14.28 \%$ of the total of projects presented in the triennium and only one project was approved. Conclusion: We conclude, therefore, that there is a shortage of projects, both presented and approved, involving this theme. In this way, it is sought to encourage the Third Sector institutions that act in the provision of oncological services to participate in the program through projects with technical operational capacity, considered of relevance to the Unified Health System and its users.

Key words: Medical Oncology; National Health Programs; Health Human Resource Training; Pediatrics.
\end{abstract}

Resumen

Introducción: El Programa Nacional de Apoyo a la Atención Oncológica (Pronon) es fruto de una asociación entre los Ministerios de Salud y de la Hacienda, que, conjuntamente, determinan un valor global de renuncia fiscal a ser empleada a cada período. Entre los temas prioritarios del programa, se tiene la oncología infantil y juvenil y es posible la presentación de proyectos, por las instituciones de salud del Tercer Sector, en las áreas de asistencia, investigación y formación profesional. Objetivo: Presentar un panorama del trienio 2016-2018 referente a los proyectos dirigidos a esa temática, en el área de formación, entrenamiento y perfeccionamiento de recursos humanos. Método: Estudio de caso y análisis cuantitativo, teniendo como muestra todos los proyectos presentados al Ministerio de Salud, en el ámbito del Pronon, en el período. Resultados: Se pudo observar que el número de proyectos presentados en el marco del Pronon en la temática de oncología infantil y juvenil es muy bajo, representando el 14,28\% del total de proyectos presentados en el trienio y sólo un fue aprobado. Conclusión: Se concluye, por lo tanto, que hay una carencia de proyectos, tanto presentados como aprobados, involucrando esta temática. De esta forma, se busca incentivar a las instituciones del Tercer Sector que actúan en la prestación de servicios oncológicos a participar del programa por medio de proyectos con capacidad técnica operacional, considerados de relevancia para el Sistema Único de Salud y sus usuarios.

Palabras clave: Oncología Médica; Programas Nacionales de Salud; Capacitación de Recursos Humanos en Salud; Pediatría.

\footnotetext{
${ }^{1}$ Universidade de Brasília (UnB). Brasília (DF), Brasil. Orcid iD: https://orcid.org/0000-0002-6393-1641.

${ }^{2}$ Universidade Federal de Juiz de Fora (UFJF). Juiz de Fora (MG), Brasil. Orcid iD: https://orcid.org/0000-0002-0678-8295.

Endereço para correspondência: Vanessa Murta Rezende. SRTV 702, Via W 5 Norte, Asa Norte - Edifício PO 700 - $4^{\circ}$ andar. Brasília (DF), Brasil. CEP $70719-000$. E-mail:vmrezende@gmail.com.
} 


\section{INTRODUÇÃO}

O Programa Nacional de Apoio à Atenção Oncológica (Pronon) ${ }^{1}$ pertence ao Governo Brasileiro e é protagonizado pelos Ministérios da Saúde e da Fazenda, visando ao incentivo de açóes e serviços promovidos por entidades, associaçóes e fundaçóes privadas sem fins lucrativos que atuam no campo da oncologia.

Para Di Pietro ${ }^{2}$, as parcerias, em sentido amplo, designam todas as formas de sociedade que, sem formar uma nova pessoa jurídica, são organizadas entre os setores públicos e privados para consecução de fins de interesse público. No caso do Pronon, essa parceria ocorre entre a Administraçáo Pública e as Instituiçôes de Saúde do Terceiro Setor.

Instituído pela Lei $\mathrm{n}^{\circ} .12 .715 / 2012^{3}$, regulamentada pelo Decreto n ${ }^{0} .7 .988 / 2013^{4}$ e atualizado pela Portaria de Consolidação MS no . 05/20175 , anexo LXXXVI, que revogou a Portaria GM/MS no. $1.550 / 14^{6}$, o programa é financiado por recursos de renúncia fiscal, cujo montante é definido formalmente pelos Ministérios partícipes entre três vertentes:

1. Ampliação da oferta de serviços e expandir a prestação de serviços médico-assistenciais.

2. Apoio na formaçáo, o treinamento e o aperfeiçoamento de recursos humanos - em todos os níveis.

3. Realizaçãodepesquisasclínicas,epidemiológicas, experimentais e socioantropológicas.

A legislação que versa sobre o programa delimita áreas prioritárias sobre as quais as instituiçôes devem apresentar projetos destinados à capacitação dos profissionais da área da saúde, preferencialmente, vinculados ao Sistema Único de Saúde (SUS).

Conforme o artigo $6^{\circ}$ da Portaria de Consolidação no. 05/2017, anexo LXXXVI, entre as áreas prioritárias para açóes e serviços na área oncológica, tem-se o:

IV- Desenvolvimento de projetos de educação permanente e formaçáo de recursos humanos direcionados a profissionais que atuem na área de saúde em todos os níveis de atenção, especialmente [...] d) voltados para a educação permanente na área de oncologia pediátrica (p. 5) .

Com base nos dados do Instituto Nacional de Câncer José Alencar Gomes da Silva (INCA), para o biênio 201820197, estimam-se, para o Brasil, 12.500 novos casos de câncer infantojuvenil, correspondendo a 3\% do total de novos casos de neoplasias no país. Atualmente, o índice de cura dos casos de neoplasia infantil diagnosticados no Brasil é considerado alto (80\%), embora seja a segunda causa de óbito na faixa etária de 0 a 19 anos.
Conforme dados técnicos publicados na literatura da área, "estima-se que em torno de $70 \%$ das crianças acometidas de câncer podem ser curadas se o diagnóstico for precoce e a doença tratada em centros especializados" ${ }^{\text {". }}$.

No território, entre as açôes que devem ser realizadas pela gestão da saúde, é primordial que se faça o monitoramento da morbimortalidade por câncer, de modo a obterem-se informaçóes e instrumentos para o estabelecimento de iniciativas de prevenção, controle do câncer e de seus fatores de risco.

Concomitantemente às açôes assistenciais e de gestão, as ações de formação e capacitação dos profissionais de saúde, que desenvolvem suas ações de cuidado de pacientes oncológicos pediátricos, devem ser um dos pilares para o incremento de qualidade do serviço prestado, o que garantirá que eles possam atuar, com maior propriedade, responsabilidades e humanidade nos tratamentos de crianças e jovens, em uma área tâo importante e sensível que é a oncologia.

Conforme Lacombe (p. 14) ${ }^{9}$, é de fundamental importância:

selecionar, formar, integrar e aperfeiçoar um grupo de pessoas para trabalhar numa empresa como uma verdadeira equipe, com objetivos definidos, fazendo com que cada membro conheça seu papel, coopere com os demais e "vista a camisa" para produzir resultados [...].

Por meio da aprendizagem contínua, e, nesse contexto, tem-se a educação permanente na saúde. E desejável que os profissionais desenvolvam conhecimento técnico, nos âmbitos biológico, psicológico e emocional, contemplando a prática multiprofissional no sentido do aprimoramento do cuidado oncológico, principalmente o infantojuvenil.

Em estudo feito sobre o diagnóstico precoce do câncer infantojuvenil na atenção primária ${ }^{10}$, concluiu-se que há "necessidade de aprimorar a formação dos profissionais de saúde que atuam no cuidado à criança e adolescente na atenção primária à saúde, privilegiando a abordagem do tema diagnóstico precoce do câncer infantojuvenil".

Nesse mote, é esperado que as instituiçôes oncológicas pediátricas tenham buscado, por meio do Pronon, recursos estratégicos para formar, capacitar e aperfeiçoar os conhecimentos dos profissionais da área da saúde.

No Ministério da Saúde, a Coordenação-Geral de Ações Técnicas em Educação na Saúde do Departamento de Gestão da Educação na Saúde da Secretaria de Gestão do Trabalho e da Educação na Saúde (CGATES/ DEGES/SGTES/MS) é a área responsável pela análise, acompanhamento e monitoramento dos projetos do Pronon e busca incentivar o desenvolvimento e a aplicabilidade de projetos na área oncológica, principalmente na vertente infantojuvenil, visando a suprir 
a carência de projetos nessa área e o fortalecimento das instituiçôes do Terceiro Setor envolvidos nessa temática ${ }^{11}$.

De acordo com Magalhães, Gadelha, Macedo e Cardoso:

o tema do câncer na criança necessita penetrar em todas as instâncias e agendas de saúde em nosso país, como tema de relevância máxima, para que se acelerem os avanços concretos na sobrevida da criança e do adolescente com câncer no Brasil (p. 4) ${ }^{12}$.

Dessa forma, esta pesquisa teve o objetivo de identificar, entre os projetos apresentados no âmbito do Pronon ao Ministério da Saúde, no triênio 2016-2018, os do campo de atuação de formação, treinamento e aperfeiçoamento de recursos humanos em todos os níveis, que compreendam propostas de cursos na temática de oncologia infantojuvenil, em todo o país.

\section{MÉTODO}

O estudo, de natureza transversal e descritiva, foi realizado por meio de análise dos dados relacionados aos projetos voltados para o campo de atuação na área de formação, treinamento e aperfeiçoamento de recursos humanos em todos os níveis, apresentados ao Ministério da Saúde, no triênio 2016 a 2018.

Conforme definido por sua portaria de instituição, o Pronon possui os seguintes macroprocessos, de acordo com o rito administrativo do programa estabelecido pelo Ministério da Saúde ${ }^{1}$ : Credenciamento da Instituição no Programa; Elaboração do Projeto pela Instituição; Apresentação do Projeto ao Ministério da Saúde; Análise Técnica do Projeto (aprovação ou reprovação do Projeto); Validação do Comitê Gestor do Programa; Monitoramento; Encerramento e Prestação de Contas.

A partir do recebimento do projeto pelo Ministério, é iniciado o monitoramento desses projetos: os documentos ingressam por meio do Sistema Eletrônico de Informação (SEI) e os dados são transcritos para uma planilha de monitoramento. Essa planilha contém os dados essenciais do projeto, quais sejam: nome da instituição, nome do projeto, temática principal dos cursos propostos, público-alvo, período de execução, valor da proposta, região brasileira, município, Estado, se os cursos atendem à região de vazio assistencial, se a área técnica é favorável ou não à execuçáo do projeto.

Após o levantamento de todos os projetos e por meio de filtros aplicados nas variáveis: temática dos cursos; UF, público-alvo, foram identificados os que apresentaram proposta de formação, treinamento e recursos humanos em todos os níveis, sobre a temática oncologia infantojuvenil.

A coleta de dados foi realizada considerando somente o triênio 2016-2018, e, a partir da consolidação dos dados, foram identificadas a distribuição geográfica dos projetos, as áreas prioritárias contempladas, as áreas náo contempladas, bem como uma análise comparativa dos projetos apresentados pelas instituiçóes e os efetivamente aprovados pelo Ministério da Saúde, após a análise técnica.

\section{RESULTADOS}

Após a coleta e sistematização dos dados sobre os projetos voltados para a área de formação, treinamento e recursos humanos em todos os níveis no âmbito do Pronon, observou-se que, no triênio 2016-2018, o Ministério da Saúde recebeu 63 propostas de projetos na área da oncologia.

É possível observar que, no período contemplado pelo estudo, e considerando a relevância do tema no país, o quantitativo de projetos apresentados no âmbito do Pronon, na temática de oncologia infantojuvenil, é muito baixo, representando apenas $14,28 \%$ do total de projetos expostos no triênio.

Observa-se que, dos nove projetos apresentados, apenas um, da Região Sudeste, foi considerado apto para iniciar a execução, conforme mostrado no Gráfico 1.

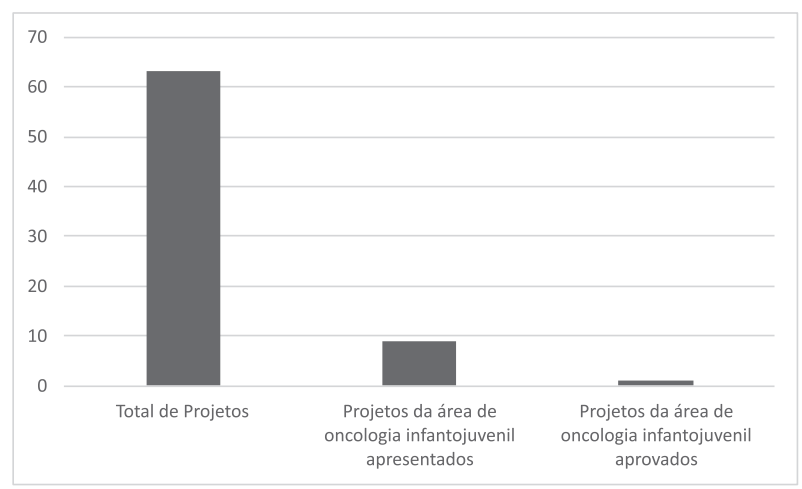

Gráfico 1. Comparativo de Projetos (número absoluto de projetos apresentados). Triênio 2016-2018

Fonte: Sistema Eletrônico do Serviço de Informaçôes ao Cidadão (e-SIC). [Internet]. Versão 3.1.0. Brasília: Ministério da Saúde. C2013 - [acesso 2018 Ago 29]. Disponível em: http://portalms.saude.gov.br/acesso-a-informacao/ servico-de-informacao-ao-cidadao.

Gráfico adaptado pelas autoras.

Ressalta-se que o Ministério da Saúde consolidou os dados de 2016 e 2017, não sendo possível neste estudo, portanto, separar as informaçóes por ano. Dessa forma, os resultados serão apresentados em dois períodos: ciclo 2016-2017 e ciclo 2018.

\section{CICLOS 2016-2017}

Constatou-se que, no período 2016-2017, foram apresentados ao Ministério da Saúde 35 projetos na área de formação, treinamento e aperfeiçoamento em todos os níveis no âmbito do Pronon. Entre eles, somente quatro projetos voltados para a área de oncologia infantojuvenil. 
Apenas três Estados apresentaram projetos, conforme demonstra o Gráfico 2.

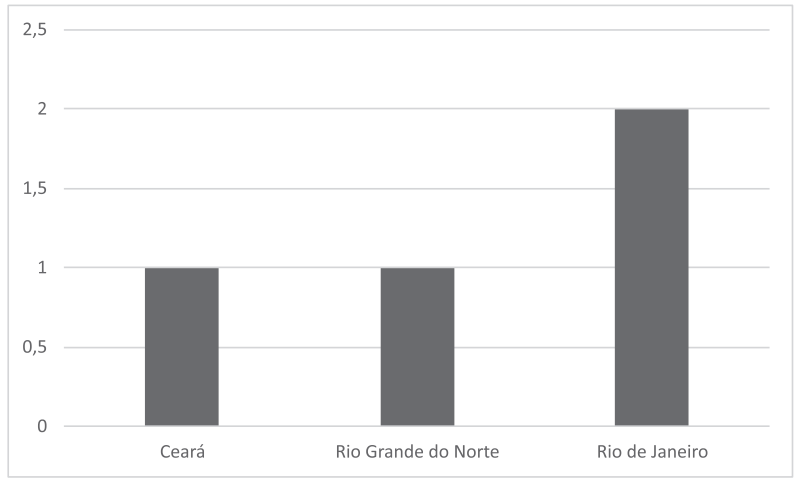

Gráfico 2. Número absoluto de projetos na temática oncologia infantojuvenil, por unidade federativa, apresentados no período 2016-2017

Fonte: Sistema Eletrônico do Serviço de Informaçóes ao Cidadāo (e-SIC). [Internet]. Versão 3.1.0. Brasília: Ministério da Saúde. C2013 - [acesso 2018 Ago 29]. Disponível em: http://portalms.saude.gov.br/acesso-a-informacao/ servico-de-informacao-ao-cidadao.

Desses projetos submetidos à análise, ressalta-se que somente dois obtiveram indicativo de mérito favorável pela área técnica. Contudo, um deles não foi aprovado por não ser prioritário, com base no critério de vazio assistencial. Assim, no período de 2016-2017, apenas um projeto na área de oncologia pediátrica foi considerado apto para execução.

\section{CICL0 2018}

Em 2018, foram apresentados 28 projetos, e, desses, cinco tinham a temática voltada para oncologia infantojuvenil, oriundos de quatro Estados, conforme demonstra o Gráfico 3.

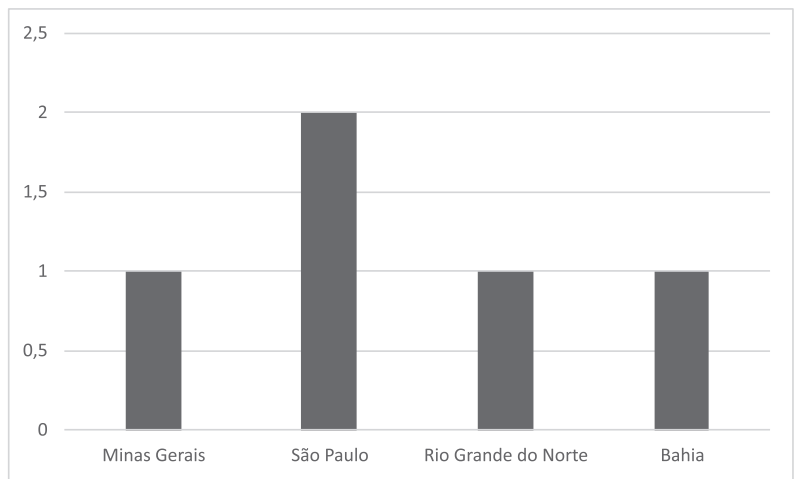

Gráfico 3. Número absoluto de projetos apresentados na área de formação, treinamento e recursos humanos a todos os níveis referentes à temática oncologia infantojuvenil no ciclo 2018 por Estados brasileiros

Fonte: Sistema Eletrônico do Serviço de Informaçóes ao Cidadão (e-SIC). [Internet]. Versão 3.1.0. Brasília: Ministério da Saúde. C2013 - [acesso 2018 Ago 29]. Disponível em: http://portalms.saude.gov.br/acesso-a-informacao/ servico-de-informacao-ao-cidadao.
Dos projetos apresentados, nenhum obteve o indicativo de mérito favorável pela área técnica. Também foi constatado que somente o Estado do Rio Grande do Norte submeteu projetos nos dois períodos, o que indica que a maioria das Instituiçôes que tiveram seus projetos com julgamento de mérito desfavorável não desenvolveu novos projetos no ano seguinte.

\section{CONCLUSÃO}

Segundo os dados referentes aos projetos apresentados no Programa Pronon, no triênio 2016-2018, é possível concluir que, na área de formação, treinamento e aperfeiçoamento em todos os níveis, há uma carência de propostas de projetos apresentados e aprovados na temática de oncologia infantojuvenil.

No montante de 63 projetos apresentados, somente nove foram destinados ao tema oncologia infantojuvenil; destes, apenas um projeto, de 2017 , encontra-se apto à execução.

Contudo, por meio do Pronon, espera-se que o Ministério da Saúde contribua cada vez mais fortemente no combate ao câncer infantojuvenil no Brasil, proporcionando ao Terceiro Setor uma oportunidade de oferecer à população saúde pública qualificada, principalmente nas regióes menos favorecidas, onde há o vazio assistencial.

É de extrema importância para que se amplie, tanto quantitativamente como qualitativamente, a apresentação e aprovação de projetos de formação, capacitação e qualificação em todos os níveis que versam sobre a temática oncologia infantojuvenil, de forma a contribuir para o aperfeiçoamento dos profissionais de saúde que atuam nessa área.

Quanto à não aprovação dos poucos projetos apresentados nessa temática, sugere-se que sejam feitos novos estudos para identificar as causas da não aprovação, que podem estar relacionadas, por exemplo, à não conformidade com a legislação ligada ao programa ou à inadequação do projeto aos critérios estabelecidos pelo Ministério da Saúde.

\section{CONTRIBUIÇÕES}

Ambas as autoras contribuíram substancialmente na concepção ou no planejamento do estudo; na obtenção, na análise e/ou interpretação dos dados; assim como na redação e/ ou revisão crítica e aprovação final da versão publicada.

\section{DECLARAÇÃO DE CONFLITO DE INTERESSES}

Nada a declarar. 


\section{FONTES DE FINANCIAMENTO}

Não há.

\section{REFERÊNCIAS}

1. Ministério da Saúde (BR). PRONON e PRONAS/ PCD. [Internet]. Brasília, DF: Ministério da Saúde; c2013-2018 [acesso 2018 Maio 2]. Disponível em: http://portalms.saude.gov.br/acoes-e-programas/prononpronas.

2. Di Pietro MSZ. Parcerias na administração pública: concessão, permissão, franquia, terceirização e outras formas. 4. ed. São Paulo: Atlas; 2002.

3. Presidência da República (BR). Lei no 12.715, de 17 de setembro de 2012. Altera a alíquota das contribuiçôes previdenciárias sobre a folha de salários devidas pelas empresas que especifica; institui o Programa de Incentivo à Inovaçáo Tecnológica e Adensamento da Cadeia Produtiva de Veículos Automotores [...]. Diário Oficial da União. 2012 set. 18; Seção 1. p. 1.

4. Presidência da República (BR). Decreto $\mathrm{n}^{\circ}$ 7.988, de 17 de abril de 2013. Regulamenta os arts. $1^{\circ}$ a 13 da Lei no 12.715, de 17 de setembro de 2012, que dispóem sobre o Programa Nacional de Apoio à Atenção Oncológica PRONON e o Programa Nacional de Apoio à Atençáo da Saúde da Pessoa com Deficiência - PRONAS/PCD. Diário Oficial da União. 2013 abr. 18; Seção 1. p. 2.

5. Ministério da Saúde (BR). Portaria de Consolidação no 5, de 28 de setembro de 2017. Consolidação das normas sobre as açóes e os serviços de saúde do Sistema Único de Saúde. Diário Oficial da União. 2017 out. 3. Seção I, Supl. 190, p. 360. Anexo LXXXVI.

6. Ministério da Saúde (BR). Portaria no 1.550, de 29 de julho de 2014. Redefine as regras e os critérios para o credenciamento de instituiçôes e para apresentação, recebimento, análise, aprovação, execuçấo, acompanhamento, prestação de contas e avaliação de resultados de projetos no âmbito do Programa Nacional de Apoio à Atençáo Oncológica (PRONON) e do Programa Nacional de Apoio à Atenção da Saúde da Pessoa com Deficiência (PRONAS/ PCD). Diário Oficial da União. 2014 jul. 30. Seção I, p. 37.

7. Instituto Nacional de Câncer José Alencar Gomes da Silva. Estimativa 2018: incidência de câncer no Brasil. [Internet]. Rio de Janeiro: INCA; 2017. [acesso 2018 Maio 10]. Disponível em: https://www.inca.gov.br.

8. Fermo VC, et al. O diagnóstico precoce do câncer infanto-juvenil: o caminho percorrido pelas famílias. Esc. Anna Nery [Internet]. 2014. [acesso 2018 Jun. 3];18(1):54-59. Disponível em: http://www.scielo.br/ pdf/ean/v18n1/1414-8145-ean-18-01-0054.pdf.

9. Lacombe FJM. Recursos humanos: princípios e tendências. São Paulo: Saraiva; 2005.
10. Friestino JKO, Correa CRS, Moreira Filho DC. Percepçóes dos profissionais sobre o diagnóstico precoce do câncer infantojuvenil na atençấo primária à saúde. Rev. Bras. Cancerol. [Internet]. 2017 [acesso 2018 Jun. 3];63(4):265-272. Disponível em: http://www1.inca. gov.br/rbc/n_63/v04/pdf/04-artigo-percepcoes-dosprofissionais-sobre-o-diagnostico-precoce-do-cancerinfantojuvenil-na-atencao-primaria-a-saude.pdf.

11. Ministério da Saúde (BR), Secretaria de Gestáo do Trabalho e da Educação na Saúde. Secretaria de Gestão do Trabalho e da Educação na Saúde: SGTES: políticas e açôes. 2. ed. rev. Brasília, DF: Ministério da Saúde, 2012.

12. Magalhães IQ, Gadelha MIP, Macedo CD, Cardoso TC. A oncologia pediátrica no Brasil: por que há poucos avanços? Rev. Bras. Cancerol. [Internet]. 2016 [acesso 2018 Maio 23];62(4):337-341. Disponível em: http:// www1.inca.gov.br/rbc/n_62/v04/pdf/06-artigo-opiniaoa-oncologia-pediatrica-no-brasil-por-que-ha-poucosavancos.pdf. 